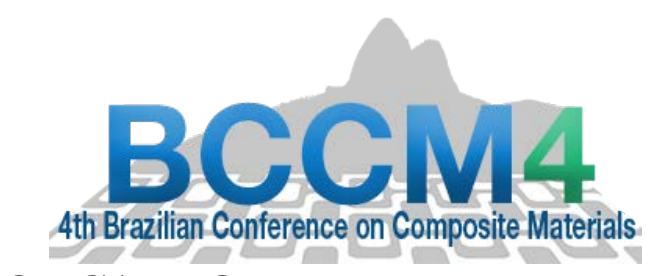

\title{
DEVELOPMENT AND CHARACTERIZATION OF CARBON FIBER REINFORCED THERMOPLASTICS - PART A: PROCESSING, THERMAL AND RHEOLOGICAL PROPERTIES
}

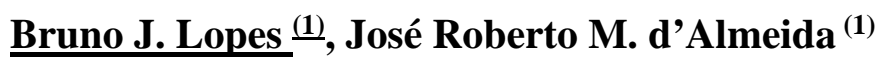 \\ (1) Department of Chemical and Materials Engineering, Pontifícia Universidade Católica do Rio \\ de Janeiro, Brazil
}

https://doi.org/10.21452/bccm4.2018.13.04

\begin{abstract}
Carbon fiber reinforced ABS (Acrylonitrile Butadiene Styrene) was produced via extrusion with varying fiber content and length. Samples were prepared from neat ABS and mixtures containing $5 \%$ and $16.7 \%$ of carbon fiber with two different initial lengths ( $3 \mathrm{~mm}$ and $6 \mathrm{~mm}$ ). In this initial study, the challenges of processing the material were investigated along with the determination of rheological and thermal properties of the final composite. For material characterization and property evaluation, Fourier-Transform Infrared Spectroscopy (FTIR), Thermogravimetric Analysis (TGA) and capillary rheology were used. Matrix degradation was observed at low processing temperatures. Results showed correlation between process additives and composite properties, mainly in thermal stability and actual fiber content. Increasing fiber content showed positive effects on thermal stability. Process additives and longer fiber lengths resulted in higher actual fiber contents. Rheological properties seemed largely unaffected mostly due to poor fiber/matrix interfaces.
\end{abstract}

Keywords: Composites; Carbon fiber; ABS; Extrusion, Additive Manufacturing.

\section{INTRODUCTION}

Much has been developed in the field of composite materials in the last decades. Researchers have found ways to make structural components faster and cheaper than ever before [1-4]. The development of thermoplastic matrix fiber reinforced composites broadens the range of applications and processing techniques for composite materials borrowing much from the knowhow developed for polymers.

The benefits of advanced fibers reinforced in common thermoplastics, such as ABS, are very attractive. These benefits are especially attractive to novel processes like additive manufacturing, which usually rely on polymers as feedstock, limiting its breadth of ability. The change to fiber reinforced composite feedstock has been already proven to revolutionize the additive manufacturing industry [5-8]. The continued development of even more capable materials will only make this revolution come faster. 
The objective of this paper was to produce carbon fiber reinforced ABS, identify the challenges of processing this composite via extrusion and characterize its thermal and rheological properties and how they vary with two different fiber contents and lengths.

\section{EXPERIMENTAL}

\subsection{Material processing}

For the initial material processing, pellets were produced mixing chopped carbon fiber (Tenax ${ }^{\circledR}-J$ HT C261, from Toho Tenax America, Inc.) in two different lengths (3 mm and $6 \mathrm{~mm}$ ) and ABS GP-35 (Terluran ${ }^{\circledR}$ GP-35, from INEOS Styrolution) at two weight percentages (\% wt). The $5 \%$ wt mixtures were initially processed as tests for setup and the $16.7 \%$ wt mixtures were processed based on previous works from Love et al. [6] and Tekinalp et al. [7] that indicates the ideal content of carbon fiber in ABS for 3D printing is between $13 \%$ and $20 \%$. In some mixtures, grape seed oil was added during mixing for improved processability, as seen in Table 1.

Each mixture was extruded using a twin-screw extruder (Leistritz ZSE $18 \mathrm{MAXX}$ ) at $200^{\circ} \mathrm{C}$, $500 \mathrm{RPM}$ and $\mathrm{kg} / \mathrm{h}$. The subsequent filaments were then water cooled and chopped to produce pellets.

For mechanical testing, filaments were extruded from each mixture's pellets using a micro extruder (Xplore Micro Compounder MC5) at 160 RPM and maximum force of $700 \mathrm{~N}$. Three different zones were used to create a temperature ladder, at $230^{\circ} \mathrm{C}$ (entry), $210^{\circ} \mathrm{C}$ and $200^{\circ} \mathrm{C}$ (exit).

Table 1: Composition of each of the produced mixtures and its nominal \% wt of fibers.

\begin{tabular}{ccccc}
\hline Mixture & ABS & Carbon Fiber (CF) & CF length & $\%$ wt CF \\
\hline I & $1000 \mathrm{~g}$ & - & - & - \\
II & $500 \mathrm{~g}$ & $25 \mathrm{~g}$ & $3 \mathrm{~mm}$ & $5 \%$ \\
III & $207.2 \mathrm{~g}$ & $10.6 \mathrm{~g}$ & $3 \mathrm{~mm}$ & $5 \%$ \\
IV & $1000 \mathrm{~g}$ & $200 \mathrm{~g}$ & $3 \mathrm{~mm}$ & $16.7 \%$ \\
V & $1000 \mathrm{~g}$ & $200 \mathrm{~g}$ & $6 \mathrm{~mm}$ & $16.7 \%$ \\
\hline
\end{tabular}

\subsection{Fourier Transform Infrared Spectroscopy (FTIR)}

The main goal with the FTIR analysis was to evaluate the possibility of degradation occurring in the polymeric matrix during processing. Initially, samples of neat ABS were extruded in two different temperatures $\left(180^{\circ} \mathrm{C}\right.$ e $\left.220^{\circ} \mathrm{C}\right)$ and the resulting pellets, tested. The data obtained was then used to define extrusion temperatures.

Afterwards, a sample from each mixture was analyzed. At this point, the objective was to determine if the presence of carbon fiber caused any abnormal effects on the materials' behavior.

All analysis were performed in a Perkin Elmer 100 FT-IR absorption spectrophotometer in the medium infrared region, with data collected in the range of 4000 to $450 \mathrm{~cm}^{-1}$ using ATR sampling at room temperature.

\subsection{Rheology}

A capillary rheometer was used to verify the effects of different contents and lengths of carbon fiber to the viscosity of the composite. 
All tests were performed using a Göttfert Rheograph 25 with capillary dimensions of $30 \mathrm{~mm}$ (length) by $2 \mathrm{~mm}$ (constant section diameter). Test conditions were of $240^{\circ} \mathrm{C}$ and shear rate ranging from 12 to $1000 \mathrm{~s}^{-1}$.

\subsection{Thermogravimetric Analysis (TGA)}

Thermogravimetric analysis served two main purposes. Firstly, it was used to determine actual carbon fiber content (\% wt) in the extruded pellets of each mixture. Secondly, using different cooling rates, it was possible to observe the decomposition kinetics occurring in each mixture at high temperature as well. Additionally, grape seed oil was also tested to evaluate its degradation at high temperatures.

For determination of actual carbon fiber content and grape seed oil degradation, a fixed cooling rate of $10^{\circ} \mathrm{C} / \mathrm{min}$. from room temperature up to $700-900^{\circ} \mathrm{C}$ under Nitrogen atmosphere was used.

For the decomposition kinetics, three cooling rates were used: 10,20 and $30^{\circ} \mathrm{C} / \mathrm{min}$. Runs were performed from $50^{\circ} \mathrm{C}$ to $750^{\circ} \mathrm{C}$, also under Nitrogen atmosphere. Then, for each mixture, a graph relating heating rates with degradation temperature at defined decomposition levels, was plotted. The decomposition levels analyzed were: 0.5\%; 1.0\%; 2.5\%; 5.0\%; $10.0 \%$ and $20.0 \%$.

In each graph, a trend line representing the best fit for the data points for each decomposition level was plotted. From each trend line, the angular coefficient was extracted via linear regression and used to calculate the activation energy for each decomposition level. This calculation used the Flynn-Wall method [9] (Equation 1), using the table presented in Doyle [10].

$$
E=\frac{-R}{b}\left[\frac{d \log \beta}{d\left(\frac{1}{T}\right)}\right]
$$

where $E$ is the activation energy $(\mathrm{J} / \mathrm{mol}), R$ is ideal gas constant $(8,314 \mathrm{~J} / \mathrm{mol} \mathrm{K}), T$ is the decomposition temperature $(\mathrm{K}), \beta$ is the heating rate $\left({ }^{\circ} \mathrm{C} / \mathrm{min}\right)$ and $b$ is a constant determined via the iterative process described in detail in [9] and [10]. The derivative term inside the brackets is the angular coefficient of the trend lines.

\section{RESULTS AND DISCUSSION}

\subsection{Matrix Degradation}

FTIR spectra showed signs of degradation of the ABS polymer at temperatures over $200^{\circ} \mathrm{C}$. This can be observed by the presence of a peak, meaning lower transmittance, in the region between $1690 \mathrm{~cm}^{-1}$ and $1800 \mathrm{~cm}^{-1}$ (Figure 1). This region is related to the stretching of $\mathrm{C}=\mathrm{O}$ bonds, typically associated with carbonyl. The presence of this group in the spectrum of the material indicates that an oxidation reaction occurred, since no oxygen is present in the molecular structure of ABS.

Based on these results, extrusion temperature was set at $200^{\circ} \mathrm{C}$ for all mixtures. This temperature was set as a compromise between polymer degradation and viscosity, to make mixing of all components possible inside the extruder with few property losses.

Further analysis of the spectra showed that neither fiber content nor length significantly influenced degradation of the matrix. 


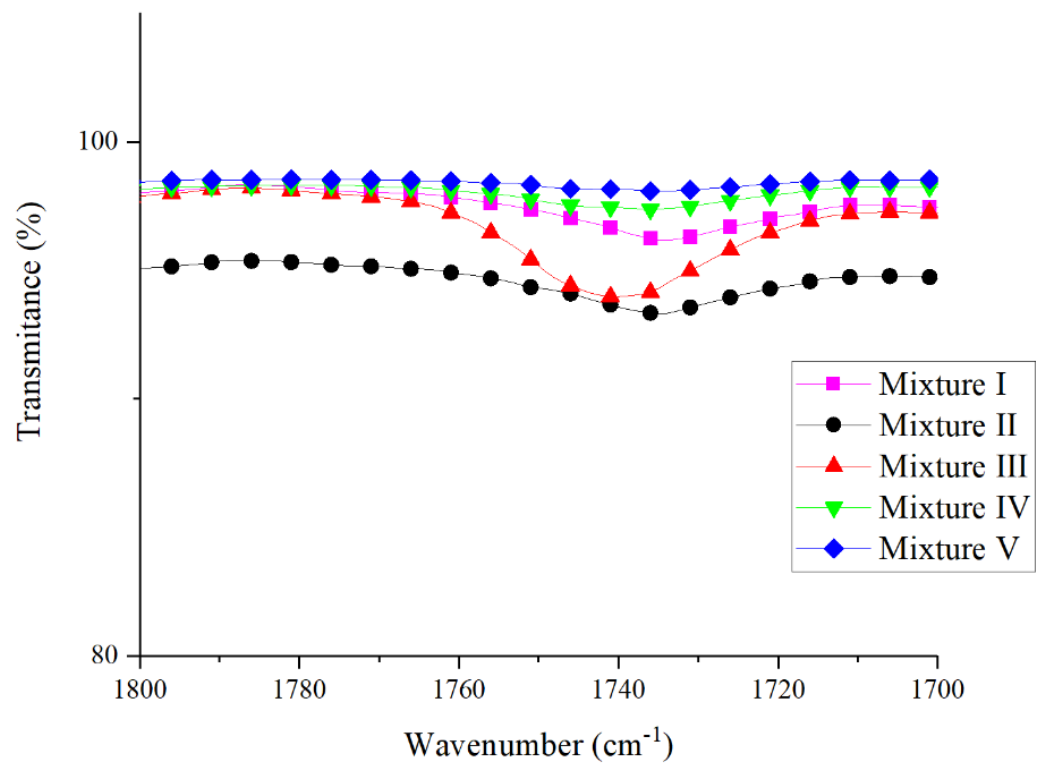

Figure 1: Comparison between mixtures in the range of the spectra associated with degradation

\subsection{Thermogravimetric Analysis}

Table 2 shows the results for the thermogravimetric analysis performed on each sample, including the grape seed oil. Results show no significant variation between mixtures but shows that the grape seed oil has lower onset and temperature at the inflection point, indicating a lower degradation threshold.

Table 2: Results of the TGA analysis for the mixtures and the grape seed oil

\begin{tabular}{cccccc}
\hline Mixture & $\begin{array}{c}\text { Sample weight } \\
(\mathrm{mg})\end{array}$ & $\begin{array}{c}\text { Onset X } \\
\left({ }^{\circ} \mathrm{C}\right)\end{array}$ & $\begin{array}{c}\text { Onset Y } \\
(\%)\end{array}$ & $\begin{array}{c}\text { Temperature at } \\
\text { inflection point }\left({ }^{\circ} \mathrm{C}\right)\end{array}$ & $\begin{array}{c}\text { Rate at the inflection } \\
\text { point }(\% / \mathrm{min})\end{array}$ \\
\hline I & 22.313 & 419.36 & 976.672 & 469.98 & -155.786 \\
II & 20.773 & 426.15 & 970.366 & 476.17 & -149.419 \\
III & 12.632 & 419.37 & 961.493 & 450.95 & -141.667 \\
IV & 17.874 & 420.94 & 979.978 & 459.2 & -131.275 \\
V & 13.801 & 428.98 & 964.327 & 474.85 & -156.269 \\
Oil & 2.18 & 399.86 & 81.722 & 428.42 & -15.426 \\
\hline
\end{tabular}

\subsubsection{Thermal Stability}

For low degradation levels, possible multiple reaction mechanisms make it inadequate to use the Flynn-Wall method for calculating the activation energy. This can be due to humidity evaporation or volatile loss during early stages of degradation. In higher degradation levels, other phenomena occur and also indicate the presence of multiple simultaneous reaction mechanisms. For these reasons, only the $5.0 \%$ and $10.0 \%$ degradation levels were used for activation energy calculations.

From the values calculated (Table 3), it is possible to observe that the thermal stability is slightly affected by the contents of the mixtures. All values are in the same order of magnitude of what can be found in the literature [11].

However, if analyzed closely, it is possible to identify a trend in activation energy variation among the five mixtures (Figure 2). Once carbon fiber is introduced, in mixture II, activation 
energy increases indicating improved thermal stability. With mixture III, which has grape seed oil in its composition, activation energy falls to its lowest. Then, with a major increase in carbon fiber \% wt, activation energy increases again, remaining relatively stable even with the longer fibers in mixture $\mathrm{V}$.

Table 3: Activation Energies for each mixture at the 5\% and 10\% degradation levels

Activation Energy $E(\mathrm{kcal} / \mathrm{mol})$

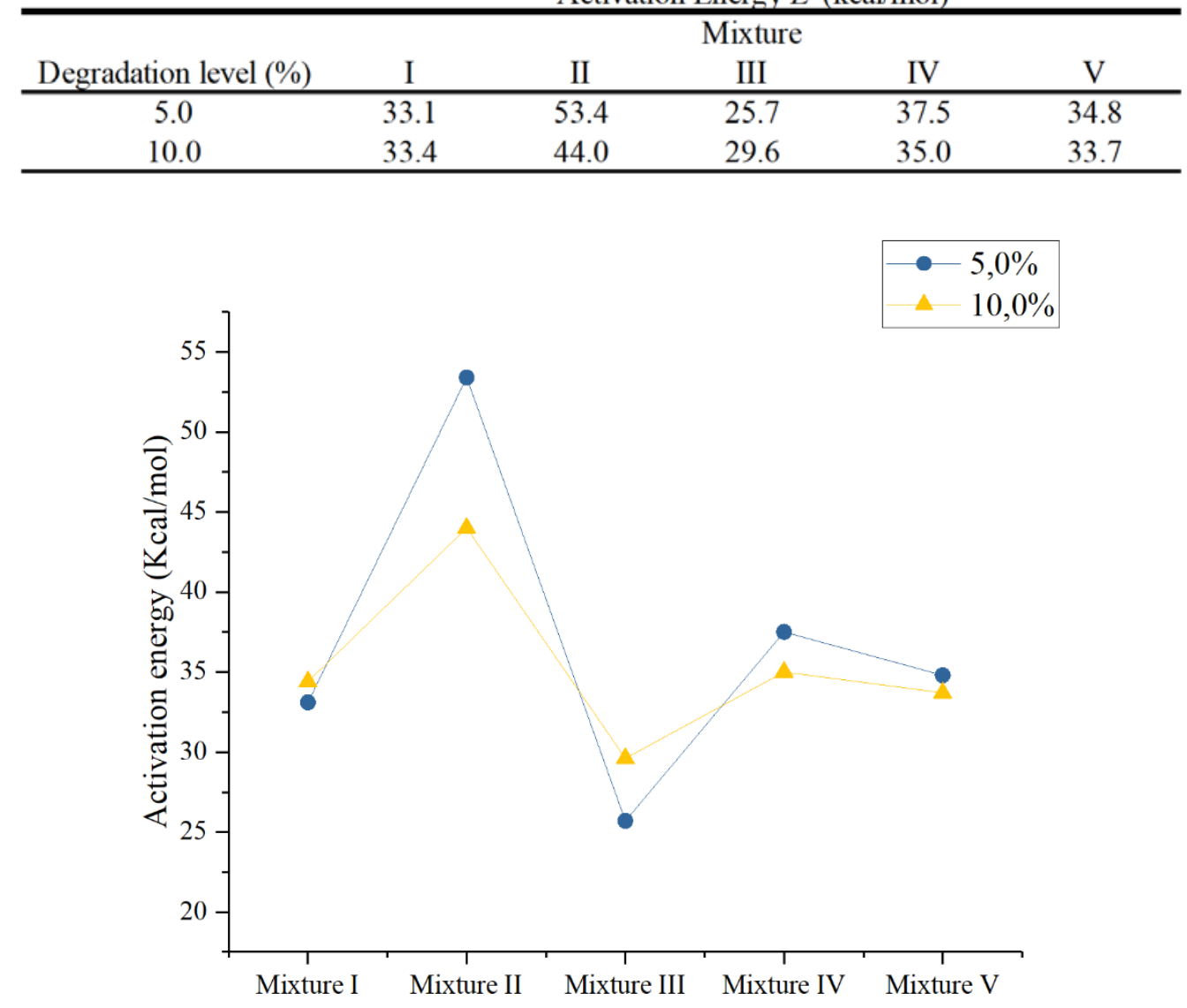

Figure 2: Activation energy plot for each mixture at the 5\% and 10\% degradation levels

\subsubsection{Actual Fiber Content}

As seen in Hull et al. [12], actual fiber content can vary from the estimated value due to process losses. The actual fiber content in each mixture was determined as the residual mass at the end of each TGA run. For neat ABS, the residual mass was zero, as seen in Figure 3, hence any remaining material can be related to the presence of carbon fiber.

It can be observed in Table 4 that the introduction of grape seed oil greatly improved the actual carbon fiber content. Fiber length also improved fiber content, with mixture $\mathrm{V}$ almost reaching its nominal value.

Mixture III showed a value higher than nominal. A possible explanation lies in the fact that the addition of grape seed oil acted as a lubricant, loosening fibers stuck to the feeding tube of the extruder, causing more fibers to enter the mixture. 


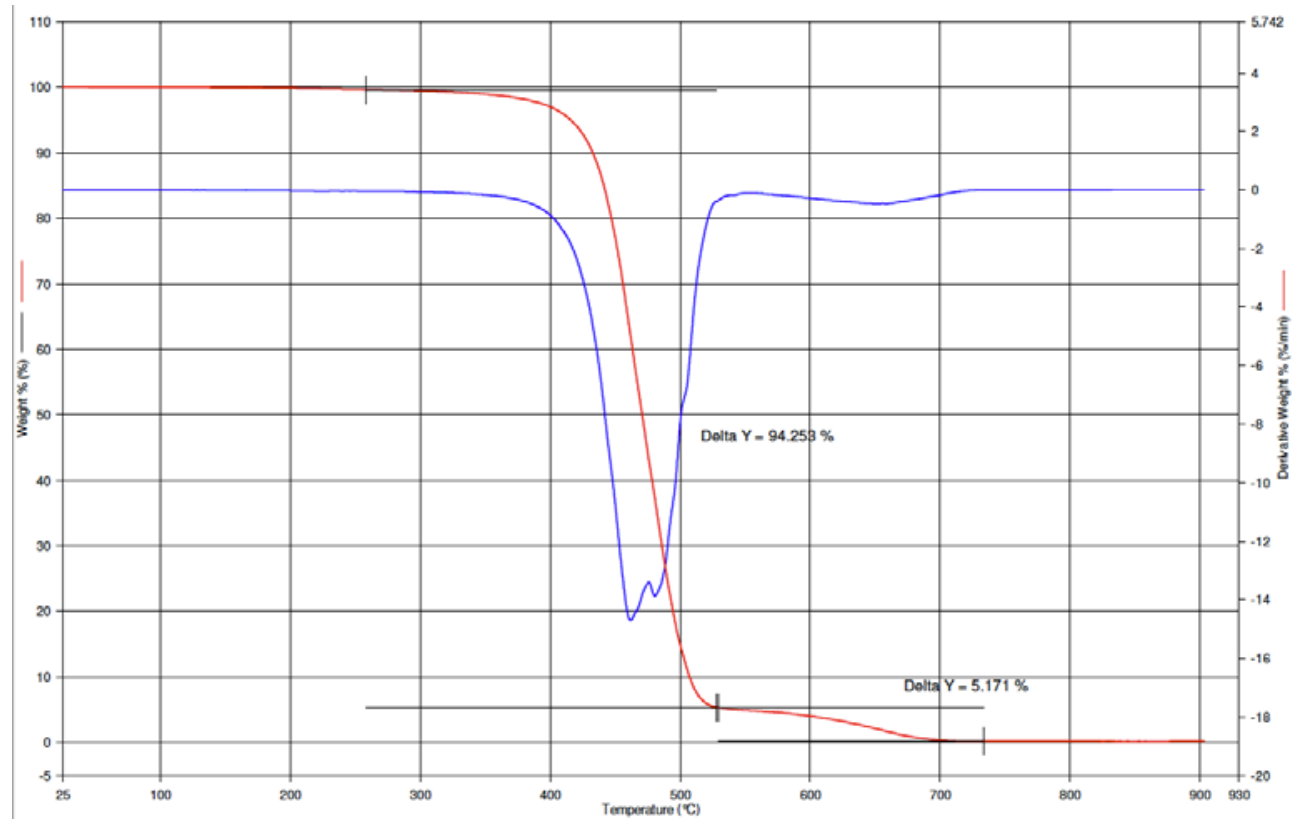

Figure 3: TGA thermogram for neat ABS showing zero residual weight

Table 4: Comparison between nominal fiber content and actual fiber content after processing for each mixture

\begin{tabular}{ccccc}
\hline Mixture & $\begin{array}{c}\text { Grape seed oil } \\
\% \text { wt }(\%)\end{array}$ & $\begin{array}{c}\text { Carbon Fiber } \\
\text { length (mm) }\end{array}$ & $\begin{array}{c}\text { Nominal \% wt CF } \\
(\%)\end{array}$ & $\begin{array}{c}\text { Actual \% wt CF } \\
(\%)\end{array}$ \\
\hline I & - & - & 0 & 0 \\
II & - & 3 & 5 & 3.1 \\
III & 1 & 3 & 5 & 5.2 \\
IV & 1 & 3 & 16.7 & 13.2 \\
V & 1 & 6 & 16.7 & 16.5 \\
\hline
\end{tabular}

\subsection{Viscosity}

From Figure 4, it is possible to observe that viscosity was largely unaffected by the presence of carbon fibers and/or grape seed oil. Small variations do occur, as expected, but they do not represent a major change in absolute viscosity value and on its behavior with increasing shear rates. Ajinjeru et al. [13] found variations up to $50 \%$ in viscosity values in similar composites. The difference in results in this case can be attributed to a poorer interface between matrix and fibers in the present material, which has been previously observed for similar materials [14]. 


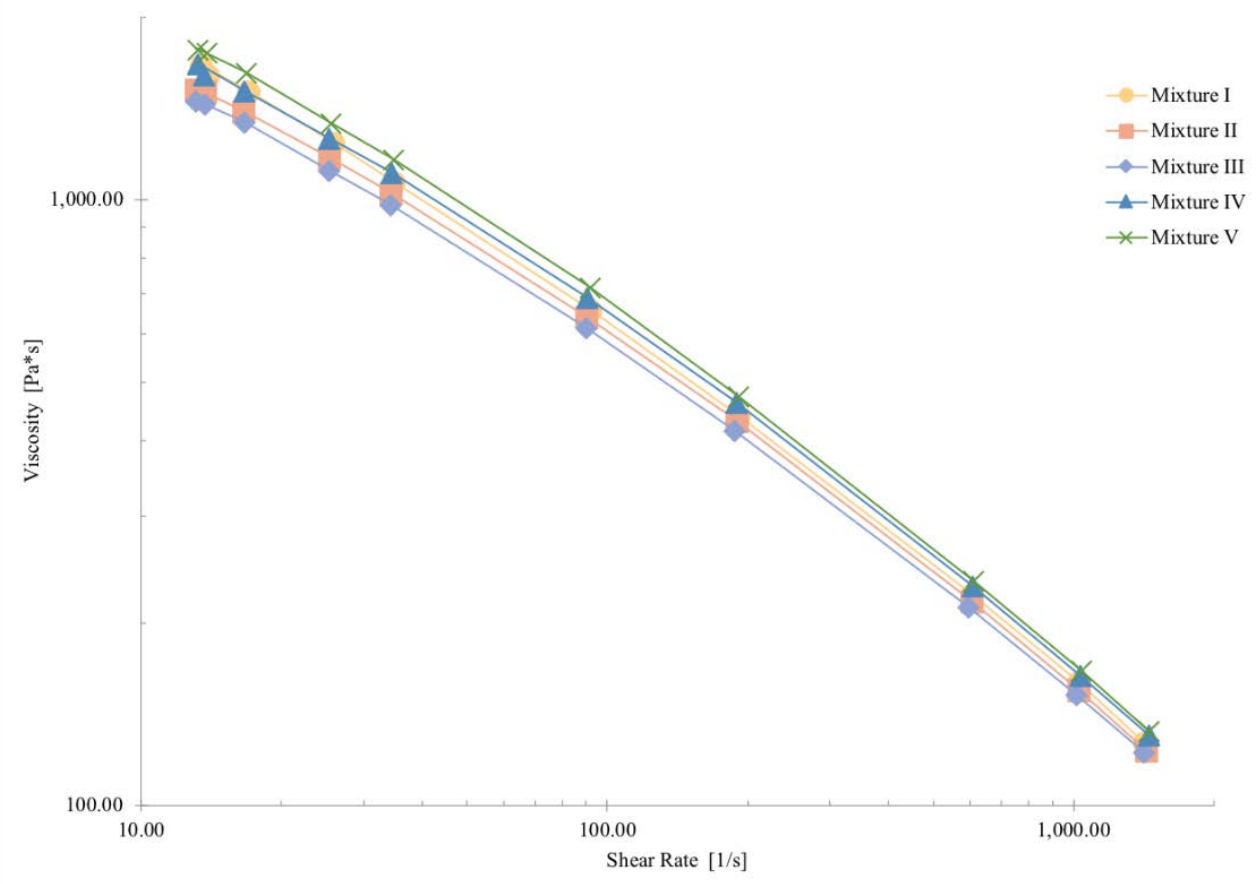

Figure 4: Graph showing the behavior of viscosity values with increasing shear rate

\section{CONCLUSIONS}

Carbon fiber reinforced ABS was successfully produced with varying fiber content and fiber length via extrusion. Degradation of the polymeric matrix was detected at temperatures as low as $220^{\circ} \mathrm{C}$ during processing, below manufacturer's recommended temperatures. Thermogravimetric analysis (TGA) showed that the composite's thermal stability is influenced by the \% wt of carbon fiber and presence of processing additives, such as the grape seed oil used. Thermal stability increased with the increase in carbon fiber content, whereas the presence of grape seed oil caused a decrease in activation energy. Also, with the aid of TGA, the actual post processing fiber content was found to be dependent on the presence of grape seed oil and fiber length, with mixtures processed with this additive and longer fibers showing higher actual fiber contents. Viscosity seemed largely unaffected by the introduction of carbon fiber, indicating a poor interface between the matrix and reinforcement phase.

From a processing perspective, results show a strong dependence of parameters such as temperature during initial compounding of the material on the final properties. Also, for mechanical mixing processes, additives that act as lubricants are paramount for avoiding waste of material and low actual fiber content in the final composite. That said, however, the properties of additives can and most likely will affect the properties of the material being processed. The negligible effect of the fiber content on the rheological behavior shows that no special consideration is needed when applying high shear rates on the composite, when compared to neat ABS.

\section{ACKNOWLEDGEMENTS}

The authors acknowledge the support from the National Council for Scientific and Technologic Development (Conselho Nacional de Desenvolvimento Científico e Tecnológico - CNPq). 
$4^{\text {th }}$ Brazilian Conference on Composite Materials. Rio de Janeiro, July $22^{\text {nd }}-25^{\text {th }}, 2018$

\section{REFERENCES}

[1] P. Feraboli, E. Peitso, F. Deleo, T. Cleveland and P. B. Stickler, 'Characterization of PrepregBased Discontinuous Carbon Fiber/Epoxy Systems,' Journal of Reinforced Plastics and Composites, vol. 28, no. 10/2009, pp. 1191-1214, May 2008.

[2] P. Feraboli, E. Peitso, T. Cleveland, P. B. Stickler and J. C. Halpin, 'Notched behavior of prepregbased discontinuous carbon fiber/epoxy systems,' Composites , vol. Part A, no. 40, pp. 289-299, March 2009.

[3] P. Feraboli, E. Peitso, T. Cleveland and P. B. Stickler, 'Modulus Measurement for Prepreg-based Discontinuous Carbon Fiber/Epoxy Systems,' Journal of Composite Materials, vol. 43, no. 19/2009, pp. 1947-1965, August 2009.

[4] P. Feraboli, F. Gasco, B. Wade, S. Maier, R. Kwan, A. Masini, L. DeOto and M. Reggiani, 'Lamborghini Forged Composite technology for the suspension arms of the Sesto Elemento,' in ASC Conference, Montreal, 2011.

[5] L. J. Love and C. Duty, 'Cincinnati Big Area Additive Manufacturing (BAAM),' Oak Ridge, TN, 2015.

[6] L. J. Love, V. Kunc, O. Rios, C. E. Duty, A. M. Elliot, B. K. Post, R. J. Smith and C. A. Blue, 'The importance of carbon fiber to polymer additive manufacturing,' J. Mater. Res., vol. 29, no. 17, 14 Sep 2014.

[7] H. L. Tekinalp, V. Kunc, G. M. Velez-Garcia, C. E. Duty, L. J. Love, A. K. Naskar, C. A. Blue and S. Ozcan, 'Highly oriented carbon fiber-polymer composites via additive manufacturing,' Composites Science and Technology, vol. 105, pp. 144-150, 16 Oct 2014.

[8] The Boeing Company, 'One For The Record Books,' 26 August 2016. [Online]. Available: http://www.boeing.com/features/2016/08/record-books-08-16.page. [Accessed 30 November 2016].

[9] J. H. Flynn and L. A. Wall, 'A quick, direct method for the determination of activation energy from thermogravimetric data,' Polymer Letters, vol. 4, no. 5, pp. 323-328, May 1966.

[10] C. D. Doyle, 'Kinetic Analysis of Thermogravimetric Data,' Journal of Applied Polymer Science, vol. V, no. 15, pp. 285-292, May/June 1961.

[11] M.-H. Yang, 'The thermal degradation of acrylonitrile-butadiene-styrene terpolymer under various gas conditions,' Polymer Testing, vol. 19, no. 1, pp. 105-110, February 2000.

[12] E. Hull, W. Grove, M. Zhang, X. Song, Z. J. Pei and W. Cong, 'Effects of Process Variables on Extrusion of Carbon Fiber Reinforced ABS Filament for Additive Manufacturing,' in ASME 2015 International Manufacturing Science and Engineering Conference, Charlotte, North Carolina, 2015.

[13] C. Ajinjeru, V. Kishore, X. Chen, J. Lindahl, Z. Sudbury, A. A. Hassen, V. Kunc, B. Post, L. Love and C. Duty, 'The Influence of Rheology on Melt Processing Conditions of Amorphous Thermoplastics for Big Area Additive Manufacturing (BAAM),' in Proceedings of the 27th Annual International Solid Freeform Fabrication Symposium - An Additive Manufacturing Conference, Austin, TX, 2016.

[14] F. Ning, W. Cong, Z. Hu and K. Huang, 'Additive manufacturing of thermoplastic matrix composites using fused deposition modeling: A comparison of two reinforcements,' Journal of Composite Materials, vol. 51, no. 27, pp. 3733-3742, 1 November 2017. 